\title{
Order convexity and concavity of Lorentz spaces
}

$$
\Lambda_{p, w}, 0<p<\infty
$$

by

\author{
Anna Kamińska (Memphis, TN) and Lech Maligranda (Luleå)
}

\begin{abstract}
We study order convexity and concavity of quasi-Banach Lorentz spaces $\Lambda_{p, w}$, where $0<p<\infty$ and $w$ is a locally integrable positive weight function. We show first that $\Lambda_{p, w}$ contains an order isomorphic copy of $l^{p}$. We then present complete criteria for lattice convexity and concavity as well as for upper and lower estimates for $\Lambda_{p, w}$. We conclude with a characterization of the type and cotype of $\Lambda_{p, w}$ in the case when $\Lambda_{p, w}$ is a normable space.
\end{abstract}

0. Introduction. The purpose of this paper is to characterize order convexity and concavity in quasi-Banach Lorentz spaces $\Lambda_{p, w}$, where $w$ is a locally integrable arbitrary weight and $0<p<\infty$. First results on this topic in Lorentz spaces belong to Creekmore [7], who has studied the spaces $L_{p, q}$ with $1<p, q<\infty$, as well as to Carothers [4] and Reisner [24], who considered the Lorentz spaces $\Lambda_{p, w}$ with a decreasing weight $w$ and $p \geq 1$. These spaces have been further investigated by the authors in [14], where convexity and concavity properties as well as the type and cotype of $\Lambda_{p, w}$ have been characterized by means of several equivalent integral inequalities as well as by indices of $w$ and its integral $W(t)=\int_{0}^{t} w$. It is well known that $\Lambda_{p, w}$ are Banach spaces whenever $w$ is decreasing and $p \geq 1([18])$. The present article is a continuation of [14]. We extend our study to $0<p$ $<\infty$ and an arbitrary weight $w$ such that $\Lambda_{p, w}$ is a quasi-Banach space. In this general setting, when the weight is not decreasing and $0<p<\infty$ is arbitrary, different methods and techniques must be used.

The paper is organized as follows. In the preliminaries we set up notations and we recall the definitions, notions and results which will be used later on. Among other results, we recall that $\Lambda_{p, w}$ is a quasi-normed space whenever

2000 Mathematics Subject Classification: Primary 46E30, 46B20; Secondary 46B42, $46 \mathrm{~B} 25$.

Key words and phrases: Lorentz spaces, quasi-Banach lattices, rearrangement invariant spaces, normability, Boyd indices, weight, weighted inequalities.

Research of L. Maligranda partially supported by a grant from the Swedish Natural Science Research Council (M-AA/MA 06857-307). 
$W$ satisfies a growth condition called $\Delta_{2}$ (see [10]). Moreover, in Theorem $\mathrm{A}$, we present several equivalent conditions for normability of $\Lambda_{p, w}$, that is, for the existence of a norm equivalent to the original quasi-norm of the space $([1,23,25])$.

The first main result, proved in the second section, states that $\Lambda_{p, w}$, $0<p<\infty$, contains an order isomorphic copy of $l^{p}$. This is an extension of the analogous result for $w$ decreasing and $p \geq 1$ due to Figiel, Johnson and Tzafriri [9]. We then apply this fact in the next sections, proving as corollaries that $\Lambda_{p, w}$ cannot be normable for $0<p<1$ and that it cannot be $r$-convex (resp. $r$-concave) for $r>p$ (resp. $r<p$ ).

In Section 3, we present criteria for $r$-convexity and for an upper $r$ estimate in $\Lambda_{p, w}$. While these criteria are different for $r \neq p$, they coincide for $r=p$. We show for instance that for $r<p, \Lambda_{p, w}$ is $r$-convex whenever the Hardy operator $H^{(r)}$ is bounded, which is equivalent to the fact that the upper Matuszewska-Orlicz index $\beta(W)$ is strictly less than $p / r$, that is, for some $\varepsilon>0, W(t) / t^{p / r-\varepsilon}$ is pseudo-decreasing. A consequence of this fact is that $\Lambda_{p, w}$ is $L$-convex ([11]). For the same $r<p, \Lambda_{p, w}$ satisfies an upper $r$-estimate if and only if $W(t) / t^{p / r}$ is pseudo-decreasing. If $r=p$, then we prove that $\Lambda_{p, w}$ is $p$-convex if and only if it satisfies an upper $p$-estimate, which is equivalent to the fact that $W(t) / t$ is pseudo-decreasing.

Section 4 contains criteria for $r$-concavity and for a lower $r$-estimate in $\Lambda_{p, w}$. While characterizations of lower $r$-estimate or $r$-concavity for $r=p$ are counterparts of the corresponding theorems in Section 3 for convexity, the integral characterization of $r$-concavity with $r \neq p$ requires more effort. We introduce here a new integral condition called $D_{p}$, which plays an analogous role in studying concavity to the role of condition $B_{p}$ in studying convexity of $\Lambda_{p, w}$. It is well known (Theorem A) that $\Lambda_{p, w}, 1<p<\infty$, is 1 -convex if and only if $w$ satisfies condition $B_{p}$, that is, for some $B>0$,

$$
\int_{x}^{\gamma} t^{-p} w(t) d t \leq B x^{-p} W(x) \quad \text { for all } x \in I,
$$

where $I=(0,1]$ or $I=(0, \infty)$, and $\gamma=1$ or $\infty$, respectively. We show (Theorem 5) that $\Lambda_{p, w}, 0<p<1$, is 1-concave if and only if $w$ satisfies condition $D_{p}$, that is, there exists $A>0$ such that

$$
\int_{0}^{x} t^{-p} w(t) d t \leq A x^{-p} W(x) \quad \text { for all } x \in I .
$$

We then apply some modifications of this condition to the study of $r$ concavity of these spaces. We conclude the paper with characterizations of type and cotype in normable $\Lambda_{p, w}$ spaces. Most results contained in this article were announced in the research report [13]. 
1. Preliminaries. We begin with some introductory definitions and results. Recall first that a quasi-Banach space $X([12])$ is a complete metrizable real vector space whose topology is given by a quasi-norm \|\| satisfying the following three conditions: $\|x\|>0, x \in X, x \neq 0 ;\|\lambda x\|=|\lambda|\|x\|, \lambda \in \mathbb{R}$, $x \in X$; and $\left\|x_{1}+x_{2}\right\| \leq C\left(\left\|x_{1}\right\|+\left\|x_{2}\right\|\right), x_{1}, x_{2} \in X$, where $C \geq 1$ is a constant independent of $x_{1}$ and $x_{2}$. Given $0<p \leq 1$, if \|\| satisfies the first two conditions and $\left\|x_{1}+x_{2}\right\|^{p} \leq\left\|x_{1}\right\|^{p}+\left\|x_{2}\right\|^{p}, x_{1}, x_{2} \in X$, then \|\| is called a $p$-norm. A quasi-Banach space $(X,\|\|)$ is said to be $p$-normable, $0<p \leq 1$, if there exists an equivalent $p$-norm in $X$. A 1 -normable space is simply called normable. If a quasi-Banach space $X$ is a vector lattice and \|\| is monotone, i.e. $\|x\| \leq\|y\|$ whenever $|x| \leq|y|$, we say that $X$ is a quasi-Banach lattice. A quasi-Banach lattice $X$ is said to be $p$-convex, $0<p<\infty$, respectively $q$-concave, $0<q<\infty$, $([8,11,17])$ if there is a constant $K>0$ such that

respectively

$$
\left\|\left(\sum_{i=1}^{n}\left|x_{i}\right|^{p}\right)^{1 / p}\right\| \leq K\left(\sum_{i=1}^{n}\left\|x_{i}\right\|^{p}\right)^{1 / p}
$$

$$
\left(\sum_{i=1}^{n}\left\|x_{i}\right\|^{q}\right)^{1 / q} \leq K\left\|\left(\sum_{i=1}^{n}\left|x_{i}\right|^{q}\right)^{1 / q}\right\|
$$

for every choice of vectors $x_{1}, \ldots, x_{n} \in X$. We also say that $X$ satisfies an upper p-estimate, $0<p<\infty$ (resp. a lower q-estimate, $0<q<\infty)$, if the above condition defining $p$-convexity (resp. $q$-concavity) is satisfied by elements $x_{1}, \ldots, x_{n} \in X$ with disjoint supports $([11,17])$. We easily observe that for $0<p \leq 1, p$-convexity implies $p$-normability and this in turn yields an upper $p$-estimate.

Given $0<p<\infty$ and a quasi-Banach lattice $X$ let $X^{(p)}=\left\{x:|x|^{p} \in X\right\}$ denote the $p$-convexification of $X$ equipped with the quasi-norm $\|x\|_{X^{(p)}}=$ $\left\||x|^{p}\right\|^{1 / p}$. It is clear that $X^{(p)}$ is 1 -convex (resp. 1 -concave) iff $X$ is $1 / p$-convex (resp. $1 / p$-concave). Notice also that a quasi-Banach space is normable iff it is 1 -convex.

Let further $I=(0,1]$ or $I=(0, \infty)$, and $L^{0} \equiv L^{0}(I,||)$ be the set of all Lebesgue measurable functions $f: I \rightarrow \mathbb{R}_{+}$, where || denotes the Lebesgue measure on $I$. By $\gamma$ we will always denote 1 in the case when $I=(0,1]$ and $\infty$ when $I=(0, \infty)$. We also agree that "decreasing" or "increasing" will mean "non-increasing" or "non-decreasing" respectively. For $f \in L^{0}$ we define its decreasing rearrangement as $f^{*}(t)=\inf \left\{s>0: d_{f}(s) \leq t\right\}, t>0$, where $d_{f}(s)=|\{t:|f(t)|>s\}|$ is the distribution function of $f$. Given $0<r<\infty$, the Hardy operators on $L^{0}$ are defined as follows:

$$
H^{(r)} f(t)=\left(\frac{1}{t} \int_{0}^{t} f^{* r}(s) d s\right)^{1 / r}, \quad H_{(r)} f(t)=\left(\frac{1}{t} \int_{t}^{\gamma} f^{* r}(s) d s\right)^{1 / r} .
$$


For $r=1, H^{(1)} f$ will be denoted by $f^{* *}$ and $H_{(1)} f$ by $f_{* *}$. It is well known $([2,16])$ that $(f+g)^{* *} \leq f^{* *}+g^{* *}$. One can also show that for any $0<r<\infty$, and $f_{1}, \ldots, f_{n} \in L^{0}$,

$$
\begin{aligned}
& H^{(r)}\left(\left(\sum_{i=1}^{n}\left|f_{i}\right|^{r}\right)^{1 / r}\right) \leq\left(\sum_{i=1}^{n}\left(H^{(r)}\left(\left|f_{i}\right|\right)\right)^{r}\right)^{1 / r}, \\
& H_{(r)}\left(\left(\sum_{i=1}^{n}\left|f_{i}\right|^{r}\right)^{1 / r}\right) \geq\left(\sum_{i=1}^{n}\left(H_{(r)}\left(\left|f_{i}\right|\right)\right)^{r}\right)^{1 / r} .
\end{aligned}
$$

The Lorentz space $\Lambda_{p, w}, 0<p<\infty$, is a subspace of $L^{0}$ such that

$$
\|f\|=\|f\|_{p, w}:=\left(\int_{I} f^{* p} w\right)^{1 / p}=\left(\int_{I} f^{* p}(t) w(t) d t\right)^{1 / p}<\infty,
$$

where a measurable weight function $w: I \rightarrow(0, \infty)$ satisfies the conditions

$$
\begin{gathered}
W(t):=\int_{0}^{t} w<\infty \quad \text { for all } t \in I, \\
\int_{0}^{\infty} w=\infty \quad \text { in the case when } I=(0, \infty) .
\end{gathered}
$$

The functional \|\|$_{p, w}$ admits several equivalent formulas $([2,4,5,16])$. In fact for $f \in \Lambda_{p, w}, 0<p<\infty$,

$$
\|f\|=\left(\int_{I} f^{* p} w\right)^{1 / p}=\left(\int_{0}^{\infty} W\left(d_{f}(t)\right) d\left(t^{p}\right)\right)^{1 / p}=\left(-\int_{I} W(t) d\left(f^{* p}(t)\right)\right)^{1 / p} .
$$

If in addition $w$ is decreasing then

$$
\|f\|=\left(\sup _{\tau} \int_{I}|f(\tau(t))|^{p} w(t) d t\right)^{1 / p},
$$

and if $w$ is increasing then

$$
\|f\|=\left(\inf _{\tau} \int_{I}|f(\tau(t))|^{p} w(t) d t\right)^{1 / p},
$$

where both the supremum and infimum are taken over all measure preserving transformations $\tau: I \rightarrow I$.

It is well known that the functional \|\|$_{p, w}$ is a norm if and only if $w$ is decreasing and $1 \leq p<\infty$ ([18]). Moreover, for any $0<p<\infty,\|\|_{p, w}$ is a quasi-norm if and only if $W$ satisfies condition $\Delta_{2}$, i.e. $W(2 t) \leq K W(t)$ for all $t \in \frac{1}{2} I$ and some $K>0$ ([10]). Indeed, if $W$ satisfies condition $\Delta_{2}$ then 
for any $f, g \in \Lambda_{p, w}$,

$$
\begin{aligned}
\|f+g\| & \leq 2^{1 / p}\left(\left(-\int_{I} W(t) d f^{* p}(t / 2)\right)^{1 / p}+\left(-\int_{I} W(t) d g^{* p}(t / 2)\right)^{1 / p}\right) \\
& \leq 2^{1 / p} K\left(\left(-\int_{I} W(t / 2) d f^{* p}(t / 2)\right)^{1 / p}+\left(-\int_{I} W(t / 2) d g^{* p}(t / 2)\right)^{1 / p}\right) \\
& \leq C(\|f\|+\|g\|) .
\end{aligned}
$$

On the other hand, assuming that \|\| is a quasi-norm and choosing $f=$ $\chi_{(0, x / 2]}$ and $g=\chi_{(x / 2, x]}$ we obtain

$$
W^{1 / p}(x)=\left\|\chi_{(0, x]}\right\| \leq C\left(\left\|\chi_{(0, x / 2]}\right\|+\left\|\chi_{(x / 2, x]}\right\|\right)=2 C W^{1 / p}(x / 2)
$$

for all $x \in I$, that is, $W$ satisfies condition $\Delta_{2}$.

Since we deal further with the functional \|\|$_{p, w}$ which is at least a quasinorm, we will assume in what follows that $W$ satisfies condition $\Delta_{2}$.

Given an arbitrary $F: I \rightarrow \mathbb{R}_{+}$we define the Matuszewska-Orlicz lower and upper indices as follows:

$$
\begin{aligned}
& \alpha(F)=\sup \left\{p \in \mathbb{R}: F(a t) \leq C a^{p} F(t)\right. \\
& \quad \text { for some } C>0 \text { and all } t \in I, 0<a \leq 1\}, \\
& \beta(F)=\inf \left\{p \in \mathbb{R}: F(a t) \leq C a^{p} F(t)\right. \\
& \quad \text { for some } C>0 \text { and all at } \in I, a \geq 1\} .
\end{aligned}
$$

If $F$ is increasing then $0 \leq \alpha(F) \leq \beta(F) \leq \infty$. A function $F: I \rightarrow \mathbb{R}_{+}$is called pseudo-increasing (resp. pseudo-decreasing) if there exists a constant $K>0$ such that $F(s) \leq K F(t)(\operatorname{resp} . F(s) \geq K F(t))$ for any $0<s<t$; $s, t \in I$. As usual two functions $F, G: I \rightarrow \mathbb{R}_{+}$are said to be equivalent if there exist positive constants $C_{1}, C_{2}$ such that $C_{1} F(t) \leq G(t) \leq C_{2} F(t)$ for every $t \in I$.

Recall also the following result due to Matuszewska and Orlicz [21].

LEMMA A $([21,16])$. If $F: I \rightarrow(0, \infty)$ is pseudo-increasing (resp. pseudo-increasing and $F$ satisfies condition $\Delta_{2}$ ) and $F(t) / t$ is pseudo-decreasing (resp. pseudo-increasing) on $I$, then there exists a positive concave (resp. convex) function on $I$ equivalent to $F$.

In the theorem below we summarize all known results characterizing normability of $\Lambda_{p, w}$.

ThEOREM A. Let $1<p<\infty$. The following conditions are equivalent.

(i) $\Lambda_{p, w}$ is normable.

(ii) The Hardy operator $f^{* *}(t)=t^{-1} \int_{0}^{t} f^{*}(s) d s$ is bounded in $\Lambda_{p, w}$.

(iii) The weight $w$ satisfies condition $S_{p}$, that is, for some $A>0$ and 
$1 / p+1 / p^{\prime}=1$

$$
\left(\int_{0}^{x} w\right)^{1 / p}\left(\int_{0}^{x}\left(\frac{1}{t} \int_{0}^{t} w\right)^{1-p^{\prime}} d t\right)^{1 / p^{\prime}} \leq A x \quad \text { for all } x \in I .
$$

(iv) The weight $w$ satisfies condition $B_{p}$, that is, there exists $B>0$ such that

$$
\int_{x}^{\gamma} t^{-p} w(t) d t \leq B x^{-p} W(x) \quad \text { for all } x \in I .
$$

(v) There is $\stackrel{x}{C}>0$ such that

$$
\int_{x}^{\gamma} t^{-p-1} W(t) d t \leq C x^{-p} W(x) \quad \text { for all } x \in I .
$$

(vi) $\beta(W)<p$, i.e. there exists $\varepsilon>0$ such that $W(x) / x^{p-\varepsilon}$ is pseudodecreasing.

Notice that Boyd proved equivalence of (ii) and (vi) in [3] when $w$ is decreasing but his techniques work just as well for general $w$ (see also [16, Ch. II, Th. 6.6]). Ariño and Muckenhoupt introduced condition $B_{p}$ and showed the equivalence of (ii) and (iv) in [1]; Sawyer proved in [25] that conditions (i)-(iv) are equivalent, and Raynaud in [23] demonstrated the equivalence of (ii) and (vi). We add here condition (v), which is an easy consequence of (iv) and integration by parts. A direct proof of the equivalence of (iv) and (vi) together with a simple proof of the fact that $B_{p}$ implies $B_{p-\varepsilon}$ was given by Maligranda in [20]. The equivalence of (v) and (vi) was proved in [19].

2. Copies of $l^{p}$ in $\Lambda_{p, w}$. We start by showing that $\Lambda_{p, w}$ contains an order copy of $l^{p}$. In the case when $w$ is decreasing and $1 \leq p<\infty$, this fact has been proved in [9] (for increasing weight see [5]; see also [6]). Notice that the proof we provide here is different than the one in [9] or [5], because of the lack of monotonicity of $w$.

Theorem 1. Let $0<p<\infty$. The Lorentz space $\Lambda_{p, w}$ contains an order isomorphic copy of $l^{p}$.

Proof. Let $I=(0,1]$ and $\varepsilon>0$. We shall construct a sequence $\left\{f_{j}\right\}_{j=1}^{\infty}$ $\subset \Lambda_{p, w}$ with disjoint supports which spans an isomorphic copy of $l^{p}$ in $\Lambda_{p, w}$. Let $k_{1}=1, \mathbb{N} \ni N_{1}>k_{1}$ and $b_{1}>0$ be such that $b_{1}^{p} W\left(2^{-k_{1}}\right)=1$ and $b_{1}^{p} W\left(2^{-N_{1}}\right)<\varepsilon / 4$. Setting

we have

$$
f_{1}=b_{1} \chi_{\left(1-2^{\left.-k_{1}, 1\right)}\right.}
$$

$$
\left\|f_{1}\right\|=1, \quad \int_{0}^{2^{-N_{1}}} f_{1}^{* p} w<\varepsilon / 4
$$


By induction we find sequences $\left(N_{j}\right)$ and $\left(k_{j}\right)$ of natural numbers and a sequence $\left(b_{j}\right)$ of positive numbers such that letting

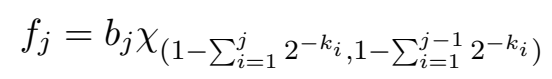

for $j=1,2, \ldots$ with $k_{1}=1$ and $\sum_{\emptyset}=0$, we have

$$
\begin{aligned}
\left\|f_{j}\right\| & =b_{j}^{p} W\left(2^{-k_{j}}\right)=1, \\
\int_{0}^{2_{j}} f_{j}^{* p} w & =b_{j} W\left(2^{-N_{j}}\right)<\varepsilon / 4^{j}, \quad k_{j}<N_{j}<k_{j+1}-1,
\end{aligned}
$$

for $j=1,2, \ldots$ It is clear that $b_{j}<b_{j+1}$ and

$$
\left(\sum_{j=1}^{\infty} f_{j}^{p}\right)^{*}=\sum_{j=1}^{\infty} b_{j}^{p} \chi_{B_{j}}
$$

where $B_{j}$ are intervals with $\left|B_{j}\right|=2^{-k_{j}}, j=1,2, \ldots$, and $\bigcup_{j=1}^{\infty} B_{j}$ is an interval $(0, \alpha)$ with $\alpha \leq 1$.

Let now $\left(a_{i}\right) \in l^{p}$ with $\left\|\left(a_{i}\right)\right\|_{p}^{p}=\sum_{i=1}^{\infty}\left|a_{i}\right|^{p}=1$. Setting $N_{0}=0$ and $C=\max \left(2^{p-1}, 1\right)$ we have

$$
\begin{aligned}
\left\|\sum_{i=1}^{\infty} a_{i} f_{i}\right\|^{p}= & \sum_{j=0}^{\infty} \int_{2^{-N_{j+1}}}^{2^{-N_{j}}}\left(a_{j+1} f_{j+1}+\sum_{i \neq j+1} a_{i} f_{i}\right)^{* p}(t) w(t) d t \\
\leq & C \sum_{j=0}^{\infty} \int_{2^{-N_{j+1}}}^{2^{-N_{j}}}\left(a_{j+1} f_{j+1}\right)^{* p}(t / 2) w(t) d t \\
& +C \sum_{j=0}^{\infty} \int_{2^{-N_{j+1}}}^{2^{-N_{j}}}\left(\sum_{i \neq j+1} a_{i} f_{i}\right)^{* p}(t / 2) w(t) d t .
\end{aligned}
$$

We will estimate separately each term of the right side of the above inequality. Since $k_{j+1}-1>N_{j}$ the following equality holds for $j=0,1, \ldots$ :

$$
P_{j+1}:=\int_{2^{-N_{j+1}}}^{2^{-N_{j}}}\left(a_{j+1} f_{j+1}\right)^{* p}(t / 2) w(t) d t=\left|a_{j+1}\right|^{p} \int_{2^{-N_{j+1}}}^{2^{-\left(k_{j+1}-1\right)}} b_{j+1}^{p} w(t) d t .
$$

By condition $\Delta_{2}$ for $W$,

$$
\int_{2^{-N_{j+1}}}^{2^{-\left(k_{j+1}-1\right)}} b_{j+1}^{p} w \leq b_{j+1}^{p} W\left(2^{-\left(k_{j+1}-1\right)}\right) \leq K b_{j+1}^{p} W\left(2^{-k_{j+1}}\right)=K,
$$

and so

$$
P_{j+1} \leq K\left|a_{j+1}\right|^{p}, \quad j=0,1, \ldots
$$


It is clear that

$$
Q_{j+1}:=\int_{2^{-N_{j+1}}}^{2^{-N_{j}}}\left(\sum_{i \neq j+1} a_{i} f_{i}\right)^{* p}(t / 2) w(t) d t \leq \int_{2^{-N_{j+1}}}^{2^{-N_{j}}}\left(\sum_{i \neq j+1} f_{i}\right)^{* p}(t / 2) w(t) d t
$$

for $j=0,1, \ldots \quad$ Moreover,

$$
\left(\sum_{i \neq j+1} f_{i}\right)^{* p}=\sum_{i=j+2}^{\infty} b_{i}^{p} \chi_{B_{i}}+\sum_{i=1}^{j} b_{i}^{p} \chi_{E_{i}}
$$

where $E_{i}$ are intervals such that $\left|E_{i}\right|=2^{-k_{i}}, i=1, \ldots, j$, and $\bigcup_{i=j+2}^{\infty} B_{i} \cup$ $\bigcup_{i=1}^{j} E_{i}$ is also an interval. As $k_{j+2}-2 \geq N_{j+1}$,

$$
\left|\bigcup_{i=j+2}^{\infty} 2 B_{i}\right| \leq 2 \sum_{i=k_{j+2}}^{\infty} 2^{-i}=2^{-\left(k_{j+2}-2\right)} \leq 2^{-N_{j+1}}
$$

whence

$$
\int_{2^{-N_{j+1}}}^{2^{-N_{j}}}\left(\sum_{i=j+2}^{\infty} b_{i}^{p} \chi_{B_{i}}\right)(t / 2) w(t) d t=0, \quad j=0,1, \ldots
$$

Now, since $N_{j}>k_{j}$ and $\left|E_{i}\right|=2^{-k_{i}}$, we have $\chi_{2 E_{i} \cap\left(0,2^{-N_{j}}\right)} \leq \chi_{\left(0,2^{-k_{j}}\right)}$, $i=1, \ldots, j$. Thus

$$
\begin{aligned}
\int_{2^{-N_{j+1}}}^{2^{-N_{j}}}\left(\sum_{i=1}^{j} b_{i}^{p} \chi_{E_{i}}\right)(t / 2) w(t) d t & \leq \sum_{i=1}^{j} \int_{0}^{2^{-N_{j}}} b_{j}^{p} \chi_{2 E_{i}}(t) w(t) d t \\
& \leq j \int_{0}^{2^{-N_{j}}} f_{j}^{* p}(t) w(t) d t<j \varepsilon / 4^{j}<\varepsilon / 2^{j} .
\end{aligned}
$$

Therefore, $Q_{1}=0$ and

$$
Q_{j+1}<\varepsilon / 2^{j}, \quad j=1,2, \ldots
$$

Combining the above inequalities, for any $\left(a_{i}\right) \in l^{p}$ with $\left\|\left(a_{i}\right)\right\|_{p} \leq 1$ we obtain

$$
\left\|\sum_{i=1}^{\infty} a_{i} f_{i}\right\| \leq(K C)^{1 / p}\left(\sum_{j=0}^{\infty}\left|a_{j+1}\right|^{p}+\varepsilon\right)^{1 / p},
$$

and thus for all $\left(a_{i}\right) \in l^{p}$ and some $M>0$,

$$
\left\|\sum_{i=1}^{\infty} a_{i} f_{i}\right\| \leq M\left\|\left(a_{i}\right)\right\|_{p}
$$


On the other hand, since $f_{j}$ have disjoint supports, for any $\left(a_{i}\right) \in l^{p}$,

$$
\begin{aligned}
\left\|\sum_{i=1}^{\infty} a_{i} f_{i}\right\|^{p} & =\sum_{j=0}^{\infty} \int_{2^{-N_{j+1}}}^{2^{-N_{j}}}\left(\left|a_{j+1} f_{j+1}\right|+\left|\sum_{i \neq j+1} a_{i} f_{i}\right|\right)^{* p} w \\
& \geq \sum_{j=0}^{\infty}\left|a_{j+1}\right|^{p} \int_{2^{-N_{j+1}}}^{2^{-N_{j}}} f_{j+1}^{* p} w,
\end{aligned}
$$

and as $N_{j}<k_{j+1}<N_{j+1}$,

$$
\int_{2^{-N_{j+1}}}^{2^{-N_{j}}} f_{j+1}^{* p} w=\int_{I} f_{j+1}^{* p} w-\int_{0}^{2^{-N_{j+1}}} f_{j+1}^{* p} w \geq 1-\varepsilon / 4^{j+1},
$$

and so

$$
\left\|\sum_{i=1}^{\infty} a_{i} f_{i}\right\| \geq(1-\varepsilon / 4)^{1 / p}\left\|\left(a_{i}\right)\right\|_{p}
$$

This completes the proof.

3. Convexity of $\Lambda_{p, w}$. In view of Theorems $\mathrm{A}$ and 1 , we obtain the following characterization of $r$-convexity of $\Lambda_{p, w}$ for $r \neq p$. Notice that conditions (i), (ii) and (iv) have also been studied in [23].

THEOREM 2. If $0<r<p<\infty$ then the following assertions are equivalent.

(i) $\Lambda_{p, w}$ is r-convex.

(ii) The Hardy operator $H^{(r)}$ is bounded in $\Lambda_{p, w}$, that is, the quasi-norms $\|f\|$ and $\left\|H^{(r)} f\right\|$ are equivalent.

(iii) The weight $w$ satisfies condition $B_{p / r}$, that is, for some $B>0$,

$$
\int_{x}^{\gamma} t^{-p / r} w(t) d t \leq B x^{-p / r} W(x) \quad \text { for all } x \in I .
$$

(iv) $\beta(W)<p / r$ or equivalently for some $\varepsilon>0, W(t) / t^{p / r-\varepsilon}$ is pseudodecreasing.

If $r>p$ then $\Lambda_{p, w}$ is not $r$-convex.

REMARK. (a) If $0<p<1$ then $\Lambda_{p, w}$ is not normable. This fact, already noticed in [10], is a consequence of Theorem 1 as well.

(b) The space $\Lambda_{p, w}, 0<p<\infty$, is $L$-convex (for the definition and consequences see [11]). Indeed, by $\Delta_{2}$-condition for $W, \beta(W)<\infty([21,19])$ and so $\beta(W)<p / r$ for some $r>0$. Hence in view of Theorem $2, \Lambda_{p, w}$ is $r$-convex and thus $L$-convex by Theorem 2.2 of [11]. 
Proposition 1. Let $0<p<\infty$. Given two weight functions $w_{1}$ and $w_{2}$, the Lorentz spaces $\Lambda_{p, w_{1}}$ and $\Lambda_{p, w_{2}}$ coincide and there exists a constant $C$ such that $C^{-1}\|f\|_{p, w_{1}} \leq\|f\|_{p, w_{2}} \leq C\|f\|_{p, w_{1}}$ for any $f$, if and only if $W_{1}$ and $W_{2}$ are equivalent.

Proof. The sufficiency follows from the formula $\|f\|=\left(-\int_{I} W d\left(f^{* p}\right)\right)^{1 / p}$. The necessity is a consequence of the equality $\left\|\chi_{(0, x]}\right\|_{p, w_{i}}^{p}=W_{i}(x), i=1,2$.

TheOrem 3. Given $0<p, r<\infty$, the following conditions are equivalent.

(i) $\Lambda_{p, w}$ satisfies an upper $r$-estimate.

(ii) $W(t) / t^{p / r}$ is pseudo-decreasing and $r \leq p$.

(iii) $W\left(t^{r / p}\right)$ is equivalent to a concave function and $r \leq p$.

If in addition $0<r<1$, then each of the above conditions is equivalent to

(iv) $\Lambda_{p, w}$ is r-normable.

Proof. By Theorem 1, $r \leq p$. Moreover, the equivalence of (ii) and (iii) is a consequence of Lemma A. In view of Kalton's result (Theorem 2.3(ii) in [11]) any $L$-convex quasi-Banach lattice is $r$-normable for $0<r<1$ iff it satisfies an upper $r$-estimate. Hence (i) is equivalent to (iv). Now it remains to show that (i) is equivalent to (ii).

Since $\left(\Lambda_{p, w}\right)^{(1 / r)}=\Lambda_{p / r, w}$, it is enough to prove that $\Lambda_{p, w}, 1 \leq p<\infty$, satisfies an upper 1-estimate iff $W(t) / t^{p}$ is pseudo-decreasing. Let us begin with the sufficiency part, taking any $\left\{f_{i}\right\}_{i=1}^{n} \subset \Lambda_{p, w}$ with disjoint supports. By Lemma A, $W^{1 / p}(t)$ is equivalent to a concave function and in view of Proposition 1 we assume without loss of generality that $W^{1 / p}$ is concave. Since also $W^{1 / p}(0)=0, W^{1 / p}$ is subadditive $([16$, p. 51$])$. Then $d_{\sum_{i=1}^{n} f_{i}}=$ $\sum_{i=1}^{n} d_{f_{i}}$, and by subadditivity of $W^{1 / p}$ and the Minkowski inequality,

$$
\begin{aligned}
\left\|\sum_{i=1}^{n} f_{i}\right\| & =\left(\int_{0}^{\infty}\left(W^{1 / p}\left(\sum_{i=1}^{n} d_{f_{i}}(t)\right)\right)^{p} d\left(t^{p}\right)\right)^{1 / p} \\
& \leq\left(\int_{0}^{\infty}\left(\sum_{i=1}^{n} W^{1 / p}\left(d_{f_{i}}(t)\right)\right)^{p} d\left(t^{p}\right)\right)^{1 / p} \\
& \leq \sum_{i=1}^{n}\left(\int_{0}^{\infty} W\left(d_{f_{i}}(t)\right) d\left(t^{p}\right)\right)^{1 / p}=\sum_{i=1}^{n}\left\|f_{i}\right\| .
\end{aligned}
$$

In order to prove the necessity, given $0<s \leq t, t \in I$, set $n=[t / s]$ and

$$
f_{i}=\chi_{\left(\frac{(i-1) t}{2 n}, \frac{i t}{2 n}\right]}, \quad i=1, \ldots, 2 n .
$$


By an upper 1-estimate for $\Lambda_{p, w}$, there exists $C>0$ such that

$$
\begin{aligned}
W^{1 / p}(t) & =\left\|\chi_{(0, t]}\right\|=\left\|\sum_{i=1}^{2 n}\left|f_{i}\right|\right\| \leq C \sum_{i=1}^{2 n}\left\|f_{i}\right\|=C \sum_{i=1}^{2 n} W^{1 / p}(t / 2 n) \\
& =2 C n W^{1 / p}(t / 2 n) \leq 2 C(t / s) W^{1 / p}(t / 2 n) \leq 2 C(t / s) W^{1 / p}(s),
\end{aligned}
$$

which means that $W(t) / t^{p}$ is pseudo-decreasing.

THEOREM 4. Given $0<p<\infty$ the following properties are equivalent.

(i) $\Lambda_{p, w}$ is $p$-convex.

(ii) $\Lambda_{p, w}$ satisfies an upper p-estimate.

(iii) $W(t) / t$ is pseudo-decreasing.

(iv) There exists a decreasing weight $w_{0}$ such that $\Lambda_{p, w}=\Lambda_{p, w_{0}}$ and \|\|$_{p, w}$ and \|\|$_{p, w_{0}}$ are equivalent.

Moreover, for $0<p \leq 1$ each of the above properties is equivalent to

(v) $\Lambda_{p, w}$ is p-normable.

Proof. Since (i) $\Rightarrow$ (ii) is obvious and (ii) $\Rightarrow$ (iii) follows from Theorem 3 , we start with (iii) $\Rightarrow$ (iv). By Lemma A, there exists a positive concave function $W_{0}$ on $I$ equivalent to $W$. Thus $W_{0}$ is absolutely continuous on $I$ and so $W_{0}(t)=\int_{0}^{t} w_{0}$ for some decreasing positive function $w_{0}$, and then in view of Proposition $1, \Lambda_{p, w}=\Lambda_{p, w_{0}}$ and \|\|$_{p, w}$ and \|\|$_{p, w_{0}}$ are equivalent. In order to prove that (iv) yields (i), we assume without loss of generality that $w$ is decreasing and applying a suitable formula for \|\|$_{p, w}$, for any finite sequence $\left\{f_{i}\right\}_{i=1}^{n} \subset \Lambda_{p, w}$ we obtain

$$
\left\|\left(\sum_{i=1}^{n}\left|f_{i}\right|^{p}\right)^{1 / p}\right\| \leq\left(\sum_{i=1}^{n} \sup _{\tau} \int_{I}\left|f_{i}(\tau(t))\right|^{p} w(t) d t\right)^{1 / p}=\left(\sum_{i=1}^{n}\left\|f_{i}\right\|^{p}\right)^{1 / p} .
$$

For $0<p \leq 1$, the immediate implications $(\mathrm{i}) \Rightarrow(\mathrm{v})$ and $(\mathrm{v}) \Rightarrow(\mathrm{ii})$ complete the proof.

4. Concavity of $\Lambda_{p, w}$. We begin the discussion of concavity of $\Lambda_{p, w}$ with a lemma which provides a number of equivalent integral conditions. Notice that the equivalence of the first three conditions does not require assuming $\Delta_{2}$ for $W$.

LEMMA 1. Let $0<p<\infty$. The following conditions are equivalent.

(i) $\alpha(W)>p$ or equivalently $W(x) / x^{p+\varepsilon}$ is pseudo-increasing for some $\varepsilon>0$. that

(ii) The weight $w$ satisfies condition $D_{p}$, that is, there exists $A>0$ such

$$
\int_{0}^{x} t^{-p} w(t) d t \leq A x^{-p} W(x) \quad \text { for all } x \in I .
$$


(iii) There is $B>0$ such that

$$
\int_{0}^{x} t^{-p-1} W(t) d t \leq B x^{-p} W(x) \quad \text { for all } x \in I .
$$

(iv) The weight $w$ satisfies condition $D_{p}^{\prime}$, that is, for some $C>0$,

$$
\int_{0}^{x}(x-t)^{p} t^{-p} w(t) d t \leq C W(x) \quad \text { for all } x \in I .
$$

If $0<p<1$, then each of the conditions (i)-(iv) is equivalent to

(v) There is $D>0$ such that

$$
\left(\int_{0}^{x} t^{-\frac{1}{1-p}} W(t)^{\frac{1}{1-p}} d t\right)^{1-p} \leq D x^{-p} W(x) \quad \text { for all } x \in I .
$$

(vi) There is $E>0$ such that

$$
\left(\int_{0}^{x} t^{-\frac{p}{1-p}} W(t)^{\frac{p}{1-p}} w(t) d t\right)^{1-p} \leq E x^{-p} W(x) \quad \text { for all } x \in I .
$$

Proof. (i) $\Rightarrow\left(\right.$ ii). Since $W\left(2^{-k} x\right) /\left(2^{-k} x\right)^{p+\varepsilon} \leq C W(x) / x^{p+\varepsilon}$ or equivalently $W\left(2^{-k} x\right) \leq C 2^{-k(p+\varepsilon)} W(x), x>0, k=0,1, \ldots$, it follows that

$$
\begin{aligned}
\int_{0}^{x} t^{-p} w(t) d t & =\sum_{k=0}^{\infty} \int_{2^{-(k+1)} x}^{2^{-k} x} t^{-p} w(t) d t \leq \sum_{k=0}^{\infty}\left(2^{-(k+1)} x\right)^{-p} \int_{2^{-(k+1)} x}^{2^{-k} x} w \\
& \leq x^{-p} \sum_{k=0}^{\infty} 2^{(k+1) p} W\left(2^{-k} x\right) \leq C 2^{p} x^{-p} \sum_{k=0}^{\infty} 2^{k p} 2^{-k(p+\varepsilon)} W(x) \\
& =C 2^{p} x^{-p} W(x) \sum_{k=0}^{\infty} 2^{-k \varepsilon}=K x^{-p} W(x) .
\end{aligned}
$$

(ii) $\Rightarrow$ (i). First we show that if $w$ satisfies $D_{p}$ then it also satisfies $D_{p+\varepsilon}$ for some $\varepsilon>0$; in fact, for all $\varepsilon<p /(A-1)$. Multiplying the inequality in (ii) by $x^{-\varepsilon-1}$ and integrating it from 0 to $r$, we obtain

$$
\int_{0}^{r} x^{-\varepsilon-1}\left(\int_{0}^{x} t^{-p} w(t) d t\right) d x \leq A \int_{0}^{r} x^{-\varepsilon-1} x^{-p}\left(\int_{0}^{x} w\right) d x .
$$

After changing the order of integration in both integrals we get

$$
\int_{0}^{r} t^{-p} w(t)\left(\int_{t}^{r} x^{-\varepsilon-1} d x\right) d t \leq A \int_{0}^{r} w(t)\left(\int_{t}^{r} x^{-\varepsilon-1} x^{-p} d x\right) d t
$$

or 


$$
\begin{aligned}
& \frac{1}{\varepsilon} \int_{0}^{r} t^{-p-\varepsilon} w(t) d t-\frac{r^{-\varepsilon}}{\varepsilon} \int_{0}^{r} t^{-p} w(t) d t \\
& \quad \leq A\left(\frac{1}{\varepsilon+p} \int_{0}^{r} t^{-p-\varepsilon} w(t) d t-\frac{r^{-p-\varepsilon}}{\varepsilon+p} \int_{0}^{r} w(t) d t\right),
\end{aligned}
$$

and applying condition $D_{p}$ gives

$$
\begin{aligned}
\left(\frac{1}{\varepsilon}-\frac{A}{\varepsilon+p}\right) \int_{0}^{r} t^{-p-\varepsilon} w(t) d t & \leq \frac{r^{-\varepsilon}}{\varepsilon} \int_{0}^{r} t^{-p} w(t) d t-\frac{A}{\varepsilon+p} r^{-p-\varepsilon} \int_{0}^{r} w \\
& \leq\left(\frac{A}{\varepsilon}-\frac{A}{\varepsilon+p}\right) r^{-p-\varepsilon} \int_{0}^{r} w
\end{aligned}
$$

Hence, if $0<\varepsilon<p /(A-1)$ then

$$
\int_{0}^{r} t^{-p-\varepsilon} w(t) d t \leq \frac{A p}{p+\varepsilon-A \varepsilon} r^{-p-\varepsilon} \int_{0}^{r} w \quad \text { for all } r \in I
$$

i.e. $w$ satisfies condition $D_{p+\varepsilon}$. Now, if $0<x<y$ then

$$
\frac{W(x)}{x^{p+\varepsilon}} \leq \int_{0}^{x} t^{-p-\varepsilon} w(t) d t \leq \int_{0}^{y} t^{-p-\varepsilon} w(t) d t \leq K y^{-p-\varepsilon} \int_{0}^{y} w=K \frac{W(y)}{y^{p+\varepsilon}} .
$$

(iii) $\Rightarrow$ (ii). Since $\int_{0}^{x} t^{-p-1} W(t) d t \geq\left(x^{-p} / 2\right) W(x / 2)$, it follows that $\lim _{x \rightarrow 0^{+}} x^{-p} W(x)=0$. Thus, integration by parts yields

$$
\begin{aligned}
\int_{0}^{x} t^{-p} w(t) d t & =\int_{0}^{x} t^{-p} d W(t)=\left.t^{-p} W(t)\right|_{0} ^{x}-\int_{0}^{x} W(t) d\left(t^{-p}\right) \\
& =x^{-p} W(x)+p \int_{0}^{x} t^{-p-1} W(t) d t \leq(1+p B) x^{-p} W(x) .
\end{aligned}
$$

(ii) $\Rightarrow$ (iii). From the inequality $x^{-p} W(x) \leq \int_{0}^{x} t^{-p} w(t) d t$ we see that $\lim _{x \rightarrow 0^{+}} x^{-p} W(x)=0$, and so by integration by parts,

$$
\int_{0}^{x} t^{-p-1} W(t) d t=\frac{1}{p}\left(\int_{0}^{x} t^{-p} w(t) d t-x^{-p} W(x)\right) \leq \frac{A-1}{p} x^{-p} W(x) .
$$

(ii) $\Leftrightarrow\left(\right.$ iv). If condition $D_{p}$ holds then

$$
\int_{0}^{x}(x-t)^{p} t^{-p} w(t) d t \leq \int_{0}^{x} x^{p} t^{-p} w(t) d t \leq A \int_{0}^{x} w
$$

On the other hand, if (iv) is satisfied then in view of $\Delta_{2}$ for $W$,

$$
C K W(x / 2) \geq C W(x) \geq \int_{0}^{x}(x-t)^{p} t^{-p} w(t) d t \geq(x / 2)^{p} \int_{0}^{x / 2} t^{-p} w(t) d t
$$

which is condition $D_{p}$. 
(i) $\Rightarrow(\mathrm{v})$. For an appropriate $\varepsilon>0$,

$$
\begin{aligned}
& \left(\int_{0}^{x} t^{-\frac{1}{1-p}} W(t)^{\frac{1}{1-p}} d t\right)^{1-p}=\left(\int_{0}^{x} t^{-\frac{1}{1-p}}\left(t^{-p-\varepsilon} W(t)\right)^{\frac{1}{1-p}} t^{\frac{p+\varepsilon}{1-p}} d t\right)^{1-p} \\
& \quad \leq c^{1-p} x^{-p-\varepsilon} W(x)\left(\int_{0}^{x} t^{\frac{p+\varepsilon}{1-p}} d t\right)^{1-p}=d x^{-p-\varepsilon} W(x) x^{\varepsilon}=d x^{-p} W(x)
\end{aligned}
$$

(v) $\Rightarrow(\mathrm{i})$. In view of condition $\Delta_{2}$ we have

$$
K^{-1} 2^{p-1} \leq 2^{p-1} W(x / 2) \leq f(x):=x^{p}\left(\int_{0}^{x} t^{-\frac{1}{1-p}} W(t)^{\frac{1}{1-p}} d t\right)^{1-p} \leq D W(x),
$$

and so $\alpha(W)=\alpha(f)$. Moreover, for sufficiently small $\varepsilon>0$,

$$
\begin{aligned}
\frac{d}{d x}\left(x^{-p-\varepsilon} f(x)\right) & \\
= & \left(-\varepsilon x^{-\varepsilon-1} \int_{0}^{x} t^{-\frac{1}{1-p}} W(t)^{\frac{1}{1-p}} d t+x^{-\varepsilon}(1-p) x^{-\frac{1}{1-p}} W(x)^{\frac{1}{1-p}}\right) \\
& \times\left(\int_{0}^{x} t^{-\frac{1}{1-p}} W(t)^{\frac{1}{1-p}} d t\right)^{-p} \\
\geq & \left(1-p-\varepsilon D^{\frac{1}{1-p}}\right) x^{-\varepsilon-\frac{1}{1-p}} W(x)^{\frac{1}{1-p}}\left(\int_{0}^{x} t^{-\frac{1}{1-p}} W(t)^{\frac{1}{1-p}} d t\right)^{-p}>0 .
\end{aligned}
$$

Therefore $\alpha(W)=\alpha(f) \geq p+\varepsilon>p$.

$(\mathrm{vi}) \Leftrightarrow(\mathrm{v})$. For $x \in I$ we have

$$
\begin{gathered}
\int_{0}^{x} t^{-\frac{p}{1-p}} W(t)^{\frac{p}{1-p}} w(t) d t \geq x^{\frac{-p}{1-p}} \int_{0}^{x} W(t)^{\frac{p}{1-p}} w(t) d t=(1-p) x^{\frac{-p}{1-p}} W(x)^{\frac{1}{1-p}}, \\
\int_{0}^{x} t^{-\frac{1}{1-p}} W(t)^{\frac{1}{1-p}} d t \geq x^{-\frac{1}{1-p}} \int_{x / 2}^{x} W^{\frac{1}{1-p}}(t) d t \geq \frac{1}{2} x^{-\frac{p}{1-p}} W(x / 2)^{\frac{1}{1-p}} .
\end{gathered}
$$

Thus integrating by parts we obtain

$$
\int_{0}^{x} t^{-\frac{p}{1-p}} W(t)^{\frac{p}{1-p}} w(t) d t=(1-p)\left(x^{-p} W(x)\right)^{\frac{1}{1-p}}+p \int_{0}^{x} t^{-\frac{1}{1-p}} W(t)^{\frac{1}{1-p}} d t,
$$

which in fact yields the equivalence of (v) and (vi).

Remark. Recall that for a r.i. quasi-Banach space $X$ over $(I,||)$, the lower and upper Boyd indices are defined as for a r.i. Banach space $([17,22])$, that is,

$$
p(X)=\sup \{p>0: \text { there exists } C>0 \text { such that }
$$

$$
\left.\left\|D_{a}\right\| \leq C a^{-1 / p} \text { for all } 0<a<1\right\},
$$


$q(X)=\inf \{q>0:$ there exists $C>0$ such that

$$
\left.\left\|D_{a}\right\| \leq C a^{-1 / q} \text { for all } a>1\right\},
$$

where $D_{a}: X \rightarrow X$ is a dilation operator defined on $I=(0, \infty)$ as $D_{a} f(t)=$ $f(a t)$, and on $I=(0,1]$ as

$$
D_{a} f(t)= \begin{cases}f(a t) & \text { for } 0 \leq t \leq \min \left(a^{-1}, 1\right), \\ 0 & \text { for } \min \left(a^{-1}, 1\right)<t \leq 1 .\end{cases}
$$

For any our weight function $w$, we have

$$
p\left(\Lambda_{p, w}\right)=p / \beta(W), \quad q\left(\Lambda_{p, w}\right)=p / \alpha(W) .
$$

We shall sketch a proof only for $I=(0, \infty)$. Let $\left\|D_{a}\right\|$ be the operator norm of $D_{a}$ on $\Lambda_{p, w}$. Then for any $a>0$,

$$
\left\|D_{a}\right\| \geq \sup _{x>0} \frac{\left\|\chi_{(0, x / a)}\right\|}{\left\|\chi_{(0, x)}\right\|}=\sup _{x>0}\left(\frac{W(x / a)}{W(x)}\right)^{1 / p} .
$$

On the other hand, since $d_{D_{a} f}(t)=a^{-1} d_{f}(t), a>0$, we get for any $f \in \Lambda_{p, w}$,

$$
\left\|D_{a} f\right\|=\left(\int_{0}^{\infty} W\left(a^{-1} d_{f}(t)\right) d\left(t^{1 / p}\right)\right)^{1 / p} \leq \sup _{x>0}\left(\frac{W(x / a)}{W(x)}\right)^{1 / p}\|f\| .
$$

These estimates yield directly the above formulas on Boyd indices.

TheOREM 5. Let $0<p<1$. The following assertions are equivalent.

(i) $\Lambda_{p, w}$ is 1-concave.

(ii) The Hardy operator $f_{* *}(t)=t^{-1} \int_{t}^{\gamma} f^{*}(s) d s$ is bounded in $\Lambda_{p, w}$.

(iii) $\alpha(W)>p$ or equivalently for some $\varepsilon>0, W(t) / t^{p+\varepsilon}$ is pseudoincreasing.

(iv) The weight $w$ satisfies condition $D_{p}$, i.e. there exists $A>0$ such that

$$
\int_{0}^{x} t^{-p} w(t) d t \leq A x^{-p} W(x) \quad \text { for all } x \in I .
$$

Proof. (i) $\Rightarrow$ (iv). For $x, y \in I$ and for any Lebesgue measurable function $f: I \rightarrow \mathbb{R}$, we have

$$
\begin{aligned}
F(y) & :=\int_{0}^{x} f^{*}(|y-t|) d t \chi_{(0, x)}(y) \\
& =\left(\int_{0}^{y} f^{*}+\int_{0}^{x-y} f^{*}\right) \chi_{(0, x)}(y) \leq 2\left(\int_{0}^{x} f^{*}\right) \chi_{(0, x)}(y),
\end{aligned}
$$

whence

$$
\|F\| \leq 2 \int_{0}^{x} f^{*}\left(\int_{0}^{x} w\right)^{1 / p}
$$


(We adopt here the convention that $\|F\|=\infty$ if $F \notin \Lambda_{p, w}$.) On the other hand, by the reverse Minkowski inequality which follows from 1-concavity of $\Lambda_{p, w}$,

$$
\|F\|=\left\|\int_{0}^{x} f^{*}(|y-t|) d t \chi_{(0, x)}(y)\right\| \geq C \int_{0}^{x}\left\|f^{*}(|y-t|) \chi_{(0, x)}(y)\right\| d t
$$

for all $x \in I$. Setting

$$
g_{t, x}(y)=f^{*}(|y-t|) \chi_{(0, x)}(y) \quad(0<t<x, x, y \in I),
$$

we obtain

$$
\left\|g_{t, x}\right\| \geq\left(\int_{0}^{x} f^{* p} w\right)^{1 / p}
$$

which follows from the formula

$$
g_{t, x}^{*}(s)= \begin{cases}f^{*}(s / 2) \chi_{(0,2 x-2 t)}(s)+f^{*}(s-x+t) \chi_{[2 x-2 t, x)}(s) & \text { if } t<x \leq 2 t, \\ f^{*}(s / 2) \chi_{(0,2 t)}(s)+f^{*}(s-t) \chi_{[2 t, x)}(s) & \text { if } x>2 t .\end{cases}
$$

Combining the above estimates we obtain

$$
\left(\int_{0}^{x} f^{* p} w\right)^{1 / p} \leq K x^{-1} \int_{0}^{x} f^{*}\left(\int_{0}^{x} w\right)^{1 / p} \quad \text { for all } x \in I
$$

for any measurable function $f$. We can rewrite this inequality, with the convention $\infty / \infty=0$, as

$$
\frac{\int_{0}^{\gamma} f^{*} w \chi_{(0, x)}}{\left(\int_{0}^{\gamma} f^{*(1 / p)} \chi_{(0, x)}\right)^{p}} \leq K x^{-p} W(x) \quad \text { for all } x \in I .
$$

Now, recall Sawyer's duality formula ([25, Theorem 1] and [15, Corollary 1.4]). If $1<r<\infty$, then for any non-negative $g \in L^{0}$ and any locally integrable function $h \geq 0$, we have

$$
\sup _{\substack{0 \leq f \\ \text { decreasing }}} \frac{\int_{0}^{\gamma} f g}{\left(\int_{0}^{\gamma} f^{r} h\right)^{1 / r}} \approx\left(\int_{0}^{\gamma}\left(\frac{\int_{0}^{t} g}{\int_{0}^{t} h}\right)^{r^{\prime}} h\right)^{1 / r^{\prime}}+\frac{\int_{0}^{\gamma} g}{\left(\int_{0}^{\gamma} h\right)^{1 / r}},
$$

where $\approx$ denotes the equivalence of both sides with constants independent of $g$ and $h$, and the convention $\infty / \infty=0$ applies. Letting in the above formula $r=1 / p, f=f^{*}, g=w \chi_{(0, x)}$ and $h=\chi_{(0, x)}$, we get for $x \in I$,

$$
\sup _{f^{*}} \frac{\int_{0}^{\gamma} f^{*} w \chi_{(0, x)}}{\left(\int_{0}^{\gamma} f^{*(1 / p)} \chi_{(0, x)}\right)^{p}} \approx\left(\int_{0}^{x} t^{-\frac{1}{1-p}} W(t)^{\frac{1}{1-p}} w(t) d t\right)^{1-p}+\frac{W(x)}{W(\gamma)^{p}} .
$$

Thus, for every $x \in I$,

$$
\left(\int_{0}^{x} t^{-\frac{1}{1-p}} W(t)^{\frac{1}{1-p}} w(t) d t\right)^{1-p} \leq K x^{-p} W(x) .
$$

This is however condition (v) of Lemma 1 and so $w$ satisfies condition $D_{p}$. 
The implication (iv) $\Rightarrow$ (iii) has been proved in Lemma 1.

(iii) $\Rightarrow$ (ii). We shall apply here Theorem 2 of [22] stating that $H_{(1)}$ is bounded on a r.i. quasi-Banach space $X$ iff $q(X)<1$. Although this theorem has been proved there only for $I=(0, \infty)$, it is easily verified also for $I=(0,1]$. Now the implication follows since $q\left(\Lambda_{p, w}\right)=p / \alpha(W)$ by the remark before the theorem.

(ii) $\Rightarrow$ (i). First we show that for any $f \in \Lambda_{p, w},\left\|f_{* *}\right\| \geq K^{-p}\|f\|$, where $K$ is the constant in condition $\Delta_{2}$. This will be done only for $I=(0,1]$. In fact, it is clear that $f_{* *}(t) \geq f^{*}(2 t) \chi_{(0,1 / 2]}(t)$ for $t \in(0,1]$. Hence

$$
\left\|f_{* *}\right\| \geq\left(\int_{0}^{1 / 2} f^{* p}(2 t) w(t) d t\right)^{1 / p}
$$

Setting $f=\chi_{(0, a)}, 0<a \leq 1$, in view of condition $\Delta_{2}$, we have

$$
\int_{0}^{1 / 2} f^{* p}(2 t) w(t) d t=\int_{0}^{a / 2} w=W(a / 2) \geq K^{-1} W(a)=K^{-1} \int_{0}^{1} f^{* p} w .
$$

Thus, by approximating $f^{*}$ by simple functions of the form $\sum \alpha_{i} \chi_{\left(0, a_{i}\right)}$, the above formula also holds for any function $f$ in $\Lambda_{p, w}$, and so $\left\|f_{* *}\right\| \geq K^{-p}\|f\|$.

Finally, we have

$$
\begin{aligned}
C\left\|\left(\sum_{i=1}^{n}\left|f_{i}\right|\right)\right\| & \geq\left\|\left(\sum_{i=1}^{n}\left|f_{i}\right|\right)_{* *}\right\| \geq\left\|\sum_{i=1}^{n}\left|f_{i}\right|_{* *}\right\| \\
& \geq \sum_{i=1}^{n}\left\|\left(f_{i}\right)_{* *}\right\| \geq K^{-p} \sum_{i=1}^{n}\left\|f_{i}\right\|,
\end{aligned}
$$

where the first inequality follows from the boundedness of $H_{(1)}$, the second from $(|f|+|g|)_{* *} \geq f_{* *}+g_{* *}$, and the third from the reverse Minkowski inequality for $0<p<1$.

The next result is a corollary of Theorems 5 and 1 . Observe that conditions (i)-(iii) have also been investigated in [23].

THEOREM 6. If $r>p>0$ then the following assertions are equivalent.

(i) $\Lambda_{p, w}$ is $r$-concave.

(ii) The Hardy operator $H_{(r)}$ is bounded in $\Lambda_{p, w}$.

(iii) $\alpha(W)>p / r$, or equivalently $W(t) / t^{p / r+\varepsilon}$ is pseudo-increasing for some $\varepsilon>0$.

(iv) The weight $w$ satisfies condition $D_{p / r}$, that is, there exists $C>0$ such that

$$
\int_{0}^{x} t^{-p / r} w(t) d t \leq C x^{-p / r} \int_{0}^{x} w \quad \text { for all } x \in I .
$$

If $0<r<p<\infty$ then $\Lambda_{p, w}$ is not $r$-concave. 
Finally, the last two theorems completing our discussion are companions of Theorems 3 and 4, respectively.

TheOREm 7. Given $0<p, r<\infty$, the following properties are equivalent.

(i) $\Lambda_{p, w}$ satisfies a lower $r$-estimate.

(ii) $W(t) / t^{p / r}$ is pseudo-increasing and $r \geq p$.

(iii) $W\left(t^{r / p}\right)$ is equivalent to a convex function.

Proof. The proof, applying the reverse Minkowski inequality for $0<p$ $\leq 1$, is analogous to that of Theorem 3 .

TheOREm 8. Given $0<p<\infty$, the following properties are equivalent.

(i) $\Lambda_{p, w}$ is p-concave.

(ii) $\Lambda_{p, w}$ satisfies a lower $p$-estimate.

(iii) $W(t) / t$ is pseudo-increasing.

(iv) There exists an increasing weight $w_{0}$ such that $\Lambda_{p, w}=\Lambda_{p, w_{0}}$ and \|\|$_{p, w}$ and \|\|$_{p, w_{0}}$ are equivalent.

Proof. The proof is analogous to the one of Theorem 4. In fact we obtain reverse inequalities. For instance, assuming that $w$ is increasing, for any $\left\{f_{i}\right\}_{i=1}^{n} \subset \Lambda_{p, w}$,

$$
\left\|\sum_{i=1}^{n}\left|f_{i}\right|^{p}\right\|=\left(\inf _{\tau} \int_{I} \sum_{i=1}^{n}\left|f_{i}(\tau(t))\right|^{p} w(t) d t\right)^{1 / p} \geq\left(\sum_{i=1}^{n}\left\|f_{i}\right\|^{p}\right)^{1 / p}
$$

which shows that (iv) yields (i).

We conclude the paper with a corollary on the type and cotype of normable $\Lambda_{p, w}$ spaces. This is a consequence of the above characterizations as well as the well known relations between type (resp. cotype) and upper estimation (resp. lower estimation) [17] (see also Theorems 11, 14 and 15 in [14]).

Corollary 1. Let $1<p<\infty$ and $w \in B_{p}$.

(a) Let $1<r<2$. Then $\Lambda_{w, p}$ has type $r$ if and only if $\alpha(W)>0, r \leq p$ and $W(t) / t^{p / r}$ is pseudo-decreasing.

(b) Let $2<r<\infty$. Then $\Lambda_{w, p}$ has cotype $r$ if and only if $r \geq p$ and $W(t) / t^{p / r}$ is pseudo-increasing.

(c) Let $p \neq 2$. Then $\Lambda_{p, w}$ has cotype 2 (resp., type 2 ) if and only if $1 \leq p<2$ and $\alpha(W)>p / 2$ (resp., $2<p<\infty$ and $\beta(W)<p / 2$ and $\alpha(W)>0)$.

(d) Let $p=2$. Then $\Lambda_{2, w}$ has cotype 2 (resp., type 2 ) if and only if $W(t) / t$ is pseudo-increasing (resp., $W(t) / t$ is pseudo-decreasing and $\alpha(W)>0$ ). 


\section{References}

[1] M. Ariño and B. Muckenhoupt, Maximal functions on classical Lorentz spaces and Hardy's inequality with weights for nonincreasing functions, Trans. Amer. Math. Soc. 320 (1990), 727-735.

[2] C. Bennett and R. Sharpley, Interpolation of Operators, Academic Press, 1988.

[3] D. W. Boyd, The Hilbert transform on rearrangement-invariant spaces, Canad. J. Math. 19 (1967), 599-616.

[4] N. L. Carothers, Symmetric structure in Lorentz spaces, Doctoral Dissertation, The Ohio State Univ., 1982.

[5] - Rearrangement invariant subspaces of Lorentz function spaces II, Rocky Mountain J. Math. 17 (1987), 607-616.

[6] N. L. Carothers and S. J. Dilworth, Subspaces of $L_{p, q}$, Proc. Amer. Math. Soc. 104 (1988), 537-545.

[7] J. Creekmore, Type and cotype in Lorentz $L_{p q}$ spaces, Indag. Math. 43 (1981), $145-152$.

[8] J. Diestel, H. Jarchow and A. Tonge, Absolutely Summing Operators, Cambridge Stud. Adv. Math. 43, Cambridge Univ. Press, 1995.

[9] T. Figiel, W. B. Johnson and L. Tzafriri, On Banach lattices and spaces having local unconditional structure, with applications to Lorentz function spaces, J. Approx. Theory 13 (1975), 395-412.

[10] A. Haaker, On the conjugate space of Lorentz space, Technical Report, Lund, 1970.

[11] N. J. Kalton, Convexity conditions for non-locally convex lattices, Glasgow Math. J. 25 (1984), 141-152.

[12] N. J. Kalton, N. T. Peck and J. W. Roberts, An F-space Sampler, London Math. Soc. Lecture Note Ser. 89, Cambridge Univ. Press, 1984.

[13] A. Kamińska and L. Maligranda, Order convexity and concavity in Lorentz spaces with arbitrary weight, Research Report, Luleå Univ. of Technology 4 (1999), 21 pp.

[14] A. Kamińska, L. Maligranda and L. E. Persson, Convexity, concavity, type and cotype of Lorentz spaces, Indag. Math. 9 (1998), 367-382.

[15] A. Kamińska and M. Mastyło, Duality and classical operators in function spaces, preprint.

[16] S. G. Kreı̆n, Yu. I. Petunin and E. M. Semenov, Interpolation of Linear Operators, Transl. Math. Monogr. 54, Amer. Math. Soc., 1982.

[17] J. Lindenstrauss and L. Tzafriri, Classical Banach Spaces II, Springer, 1979.

[18] G. G. Lorentz, On the theory of spaces A, Pacific J. Math. 1 (1951), 411-429.

[19] L. Maligranda, Indices and interpolation, Dissertationes Math. 234 (1985).

[20] - Weighted estimates of integral operators for decreasing functions, in: Proc. Internat. Conf. "Boundary Value Problems, Special Functions and Fractional Calculus" (Minsk, 1996), Belgosuniversitet, Minsk, 1996, 226-236.

[21] W. Matuszewska and W. Orlicz, On certain properties of $\varphi$-functions, Bull. Acad. Polon. Sci. Sér. Sci. Math. Astronom. Phys. 8 (1960), 439-443.

[22] S. J. Montgomery-Smith, The Hardy operator and Boyd indices, in: Lecture Notes in Pure and Appl. Math. 175, Dekker, 1996, 359-364.

[23] Y. Raynaud, On Lorentz-Sharpley spaces, Israel Math. Conf. Proc. 5 (1992), 207228.

[24] S. Reisner, A factorization theorem in Banach lattices and its application to Lorentz spaces, Ann. Inst. Fourier (Grenoble) 31 (1981), no. 1, 239-255. 
[25] E. Sawyer, Boundedness of classical operators on classical Lorentz spaces, Studia Math. 96 (1990), 145-158.

Department of Mathematical Sciences

The University of Memphis

Memphis, TN 38152, U.S.A.

E-mail: kaminska@memphis.edu

Department of Mathematics Luleå University of Technology SE-971 87 Luleå, Sweden E-mail: lech@sm.luth.se

Received May 17, 2002

Revised version April 2, 2003 